\title{
Automated data processing system for multiple small-wedge data
}

$\underline{\text { Keitaro Yamashita }}^{1}$, Kunio Hirata ${ }^{1}$, Yoshiaki Kawano ${ }^{1}$, Go Ueno ${ }^{1}$, Kazuya Hasegawa ${ }^{2}$, Takashi Kumasaka ${ }^{2}$, Masaki Yamamoto ${ }^{1}$ ${ }^{1}$ RIKEN SPring-8 Center, ${ }^{2}$ JASRI/SPring-8

$\mathrm{X}$-ray microbeam is essential for high-resolution diffraction data collection from small and weakly diffracting protein crystals. However, the radiation damage can hamper the complete data collection from a single crystal and thus the multi-crystal approach has regained the spotlight. To facilitate crystal structure analysis using many cryo-cooled microcrystals, we are constructing an automated system for data collection and data processing on BL32XU, SPring-8. Using EIGER X 9M detector (Dectris Ltd.), fast raster-scan to locate crystal positions and the subsequent small-wedge $\left(5-10^{\circ}\right)$ shutterless data collection were achieved. For raster-scan data analysis, we adapted Cheetah [Barty et al. (2014) J. Appl. Cryst., 47, 1118-1131] for the streaming mode of EIGER to perform on-the-fly spot-finding. The spot-finding result immediately appears as the 2D heatmap on GUI application SHIKA. The data processing is automated by KAMO system (https://github.com/keitaroyam/yamtbx/blob/master/doc/kamo-en.md), which includes the data processing of individual small-wedge data, grouping/clustering of isomorphous datasets, and merging with outlier rejections. KAMO utilizes XDS [Kabsch (2010) Acta Cryst., D66, 125-132] or DIALS [Waterman et al. (2013) CCP4 Newslett., 49, 13-15] for indexing and integration, and XSCALE for scaling and merging. The whole process from sample exchange to data processing was automated in ZOO system, which has already contributed to several structure analyses from many microcrystals including membrane proteins.

Keywords:

microfocus beamline, microcrystal, merging 